\title{
Efeitos cognitivos, neuropsico- lógicos e comportamentais da exposição a baixas concentrações de chumbo na infância
}

\section{Cognitive, neuropsychological, and behavioral effects of low-level lead exposure in childhood}

\author{
Edna M. Marturano ${ }^{1}$, Luciana C. S. Elias ${ }^{2}$
}

\begin{abstract}
RESUMO
O presente trabalho consiste em uma revisão bibliográfica a respeito da exposição ao chumbo em baixas concentrações e seus efeitos em crianças. Apesar do limite tolerado de chumbo no sangue, fixado pela OMS em $10 \mathrm{mg} / \mathrm{dl}$, diversos estudos transversais e longitudinais têm apontado que doses abaixo desse limite também geram consequências negativas no desenvolvimento infantil. Na primeira infância, observa-se correlação entre exposição pré-natal ao chumbo e déficits cognitivos; e correlação inversa entre níveis correntes de chumbo no sangue e desempenho cognitivo. A exposição a baixos níveis de chumbo na primeira infância também foi associada a prejuízo no desempenho cognitivo e escolar anos depois. Exposição ao chumbo na fase escolar tem sido associada a problemas no desempenho acadêmico, na atenção e em funções neuropsicológicas diversas. Estudos recentes detectaram ligação entre baixa exposição ao chumbo e problemas de comportamento na criança. A evidência acumulada indica risco de prejuízo neuropsicológico e adaptativo em crianças expostas a baixos níveis de chumbo. Os danos são maiores em concentrações muito baixas, inferiores ao nível tolerado pela OMS, indicando a necessidade de trabalhos de prevenção.
\end{abstract}

Palavras-chave: Chumbo. Inteligência. Baixo Rendimento Escolar. Comportamento Infantil.

N os idos de 1960, o limiar tolerado de con centração de chumbo no sangue (BLL Blood Lead Level) em crianças era $60 \mu \mathrm{g} / \mathrm{dl}$; esse limiar corresponde ao nível em que sintomas clínicos de intoxicação se tornam em geral evidentes nas cri- anças. Foi a partir da década seguinte que a exposição a níveis mais baixos de chumbo passou a ser investigada. Sucessivos estudos, documentando uma associação entre plumbemia abaixo de $60 \mu \mathrm{g} / \mathrm{dl}$ e QI rebaixado, contribuíram decisivamente para importan-
1. Docente. Departamento de Neurociências e Ciências do Comportamento. Faculdade de Medicina de Ribeirão Preto - USP

2. Doutora em Psicologia Clínica particular
Correspondência:

Edna M. Marturano

Departamento de Neurociências e Ciências do Comportamento. Faculdade de Medicina de Ribeirão Preto - USP. Bloco da Saúde Mental: Rua Tenente Catão Roxo, 2650 14051-140 - Ribeirão Preto/SP. telefone 1636024626 email emmartur@fmrp.usp.br.

Artigo recebido em 18/02/2009 Aprovado em 08/12/2009 
tes medidas de saúde pública, como a eliminação do chumbo da gasolina e a diminuição progressiva do limite tolerado de chumbo no sangue, fixado pela OMS em $10 \mu \mathrm{g} / \mathrm{dl}$ a partir de 1995 . Tal redução, além de proteger as crianças, possibilitou aos pesquisadores maior acesso a populações com baixos níveis de contaminação, o que resultou em um notável acúmulo de evidência sobre efeitos de baixas concentrações de chumbo no desenvolvimento. A presente revisão focaliza alguns estudos representativos dessa fase.

\section{Efeitos da exposição pré-natal e na primeira infância}

Pesquisas sobre efeitos do chumbo em bebês e crianças pequenas têm trabalhado com desenhos longitudinais, obtendo medidas sucessivas de BLL e avaliando funções psicológicas por meio de escalas padronizadas de desenvolvimento. No estudo de Boston, ${ }^{1}$ cento e setenta crianças de classe média ou médiaalta foram acompanhadas desde o nascimento até 57 meses. Crianças com nível de chumbo no cordão umbilical entre 10 e $25 \mu \mathrm{g} / \mathrm{dl}$ mostraram déficits cognitivos modestos mas persistentes no desenvolvimento mental, até a idade de 24 meses. Nesse mesmo período, BLL pós-natal não foi associado com o desenvolvimento mental das crianças. Na idade de 57 meses, a associação entre exposição pré-natal ao chumbo e desenvolvimento mental diminuiu muito na amostra como um todo, exceto entre as crianças expostas concorrentemente a níveis mais altos de chumbo $(\mathrm{BLL} \geq 10 \mu \mathrm{g} / \mathrm{dl})$. Aos 57 meses, crianças com altos níveis de exposição pré-natal compensavam melhor as perdas, se a exposição pós-natal era baixa.

No Estudo Prospectivo sobre Chumbo da Iugoslávia ${ }^{2}$ foram obtidas medidas de BLL no cordão umbilical e depois do nascimento, até a idade de 7,5 anos. Tanto a exposição pré-natal como a pós-natal correlacionaram negativamente com QI. Em crianças mexicanas acompanhadas dos seis meses aos cinco anos, ${ }^{3}$ os efeitos negativos da exposição prévia ao chumbo sobre o desenvolvimento cognitivo foram ascendentes dos três aos quatro anos e depois decaíram, o que levou os autores a suporem a existência de um período crítico para a manifestação de efeitos da exposição pós-natal precoce ao chumbo.

$\mathrm{O}$ estudo de Canfield e colaboradores ${ }^{4}$ estendeu a associação entre BLL e prejuízo intelectual a concentrações abaixo do nível de alerta da OMS. Esses autores obtiveram medidas de exposição crôni- ca ao chumbo, a partir de BLL obtido consecutivamente aos $6,12,18,24,36,48$ e 60 meses de idade. $O$ QI foi avaliado nas idades de 3 e 5 anos. BLL era baixo na amostra, com média de exposição igual a 7,4 $\mu \mathrm{g} / \mathrm{dl}$ ao longo da vida. Além de reproduzir a relação inversa entre BLL e QI, encontrada em estudos prévios, a pesquisa trouxe uma importante contribuição ao campo ao demonstrar que BLL $<10 \mu \mathrm{g} / \mathrm{dl}$ ao longo da vida está associado a prejuízo intelectual aos $3 \mathrm{e}$ aos 5 anos. O estudo mostrou também que a perda cognitiva é maior nos níveis mais baixos de BLL, um achado que havia sido antecipado em duas meta-análises. ${ }^{5,6}$

A descoberta de que o efeito do chumbo é maior em doses mais baixas foi posteriormente confirmada para outros domínios do desenvolvimento infantil, com crianças de diferentes idades, e em níveis progressivamente menores de chumbo no sangue. ${ }^{7-10}$

\section{Efeltos tardios da exposição na primeira Infâncla}

Pesquisas vinculadas ao estudo de Boston investigaram efeitos tardios da exposição precoce a baixas concentrações de chumbo. ${ }^{11,12}$ Em uma delas, um teste de QI e um teste de desempenho educacional foram aplicados, na idade de 10 anos, a 148 crianças de classe média e média-alta, com baixa exposição ao chumbo ao longo da vida. Nas análises, foram levadas em conta 13 variáveis, ambientais ou biológicas, que poderiam afetar os resultados por terem conhecida associação com desenvolvimento cognitivo. BLL mais alto aos 24 meses, mas não em outras idades, foi significativamente associado com QI mais baixo e pior desempenho escolar. Como a média de BLL aos 24 meses era $6,5 \mu \mathrm{g} / \mathrm{dl}$, os resultados desse estudo indicam que a exposição a baixos níveis de chumbo na idade de 2 anos está associada a prejuízo no desempenho cognitivo e escolar aos 10 anos. $^{11}$

Em uma coorte de 1923 crianças, encontrou-se risco aumentado para problemas de aprendizagem aos 8 anos, associado a BLL elevado no cordão umbilical (em ambos os sexos) e na dentina de dente decíduo (nas meninas). ${ }^{12}$

A possibilidade de reversão dos efeitos da exposição precoce foi examinada em um estudo prospectivo com 375 crianças australianas, seguidas até 11-13 anos. Foi investigado se o declínio de BLL, após os dois anos de idade, estaria associado à melhora 
cognitiva. Com exceção do intervalo entre as idades de 7 e 11-13 anos, em que a correlação entre ganhos de QI e declínio de BLL foi quase significativa $(\mathrm{p}=0,09)$, nenhuma outra correlação foi detectada, sugerindo que os déficits cognitivos associados à exposição ao chumbo na primeira infância são apenas parcialmente reversíveis com um subsequente declínio de BLL. ${ }^{13}$

\section{Exposição ao chumbo na fase escolar}

Dado o pico de BLL ao redor dos 2 anos e a associação entre BLL nessa idade e QI na idade escolar, associações transversais (contemporâneas) entre BLL e QI em crianças de idade escolar têm sido interpretadas como efeitos residuais de níveis mais altos de concentração de chumbo no sangue em idade mais precoce. Chen e cols ${ }^{14}$ contestaram essa interpretação com dados do Treatment of Lead-Exposed Children Study (TLC), um ensaio clínico multicêntrico com 780 crianças, em que um grupo foi tratado com succimer para redução do BLL aos 2 anos de idade. As crianças foram seguidas até 7 anos com testes de QI e medidas de BLL. Nos resultados, associações transversais ficaram mais fortes à medida que as crianças ficaram mais velhas, ao passo que a relação entre a linha de base de chumbo e o QI diminuiu. Resultados semelhantes na relação chumbo-QI foram encontrados com dados de 1.333 crianças que participaram de sete estudos longitudinais de coorte de base populacional, realizados em quatro países. ${ }^{8}$

Na fase escolar, a exposição a chumbo em baixas concentrações $(\mathrm{BLL}<10 \mu \mathrm{g} / \mathrm{dl})$ tem sido associada também a outras habilidades, como: aritmética, leitura, raciocínio não verbal, ${ }^{15}$ integração visomotora, ${ }^{7,16}$ tempo de ração, habilidades motoras finas ${ }^{7}$ e diferentes medidas de atenção. ${ }^{7,15,17}$ Em conjunto, esses estudos sugerem que o efeito de contaminação por chumbo nos anos escolares pode ser maior do que se acredita hoje.

\section{Chumbo e comportamento}

A noção de que altas concentrações de chumbo no sangue estão associadas a alterações comportamentais não é nova. Há tempos se tem conhecimento de que após a recuperação de um episódio agudo de envenenamento por chumbo as crianças se tornam inquietas, desatentas e agressivas. ${ }^{18}$ A novidade das últimas décadas é a associação entre problemas de comportamento e exposição a chumbo em baixas concentrações.

Em um estudo, a exposição ao chumbo no terceiro mês de gestação (média $8,9 \mu \mathrm{g} / \mathrm{dl}$ ) foi associada ao relato dos pais sobre delinquência e agressão no comportamento dos filhos adolescentes, e a exposição pós-natal, com pico aos 2 anos (médias entre 10 e $34 \mu \mathrm{g} / \mathrm{dl}$ ), foi associada com comportamento delinquente e anti-social relatado pelos próprios adolescentes. ${ }^{19}$ Em crianças de 8 anos, BLL no cordão umbilical não foi associado com a prevalência geral ou a natureza dos problemas emocionais e de comportamento avaliados pelo professor, porém o nível de chumbo no dente foi significativamente associado com problemas internalizantes (ansiedade, depressão, complicações somáticas) e externalizantes (agressão, comportamento delinquente). ${ }^{20}$

$\mathrm{Na}$ coorte do estudo com crianças australianas, BLL foi avaliado periodicamente desde o nascimento até a idade de 11-13 anos, obtendo-se a média de exposição ao longo da vida. Aos 11-13 anos os pais responderam ao Child Behavior Checklist - CBCL. Levando-se em conta nas análises variáveis como psicopatologia materna e QI da criança, os resultados mostraram que, para um aumento hipotético de 10 para $30 \mu \mathrm{g} / \mathrm{dl}$ em BLL vital, o risco de ter uma pontuação acima da mediana da amostra no CBCL aumentou 3,2 vezes para os meninos e 2,8 vezes para as meninas. O risco para problemas externalizantes aumentou em ambos os sexos; para problemas internalizantes, aumentou somente entre as meninas. ${ }^{21}$

Na coorte do TCL, BLL aos 2 anos não foi associado com problemas de comportamento aos 7 anos. Por outro lado, BLL aos 7 anos teve associação com diversos indicadores comportamentais avaliados por pais e professores na mesma idade. ${ }^{22}$ Os resultados foram consistentes com um efeito direto e contemporâneo do BLL sobre problemas externalizantes e dificuldades escolares, e um efeito indireto, mediado pelo QI, sobre habilidades adaptativas e problemas internalizantes.

\section{Considerações finals}

A evidência acumulada nas últimas décadas aponta para uma clara relação inversa entre BLL e funções neuropsicológicas em crianças expostas a baixos níveis de chumbo. Na atualidade, admite-se que a ação danosa do metal não se limita aos efeitos per- 
sistentes da exposição precoce, mas se estende às situações de baixa contaminação em idades posteriores. Outro achado bem estabelecido, com implicações diretas para a prevenção, é que a maioria dos danos é causada por uma concentração muito baixa de chumbo, inferior ao nível tolerado pela OMS. Os efeitos no comportamento, ainda pouco explorados, constituem um importante campo de investigação futura.
Questões metodológicas e implicações para políticas públicas têm sido objeto de discussões e debates. Ao leitor interessado em obter informações adicionais sobre o assunto, indica-se a leitura do volume 16 da revista Archives of Clinical Neuropsychology, o artigo de Binns e colaboradore ${ }^{23}$ e a entrevista com Herbert Needman publicada na revista Public Health Reports. $^{24}$

\begin{abstract}
This is a literature review about low-level lead exposure and its effects on children. Although the current World Health Organization blood level of concern stands at $10 \mathrm{mg} / \mathrm{dL}$, numerous prospective and crosssectional studies have extended the association of blood lead and developmental impairment to lower levels of lead exposure. During early childhood, there is a positive association between prenatal lowlevel lead exposure and cognitive deficits. There is also a negative correlation between current blood lead level and cognitive performance. Low-level lead exposure in early childhood predicts cognitive and academic underachievement years later. During the school years, low-level lead exposure is concurrently associated to impairments in academic achievement, attention, and several neuropsychological functions. Recent investigations have detected a relationship between lead exposure and behavior problems in children. The evidence points to the neuropsychological and adaptive risks of low-level lead exposure during early and middle childhood and suggests that there is no safety margin at existing exposures. Harm is greater in very small concentrations, below the current WHO level of concern, thus indicating the need for prevention efforts.
\end{abstract}

Keywords: Lead. Intelligence. Underachievement. Child Behavior.

\section{Referências Bibliográficas}

1. Bellinger D, Sloman J, Leviton A, Rabinowitz M, Needleman $\mathrm{HL}$, Waternaux C. Low-level lead exposure and children's cognitive function in the preschool years. Pediatrics. 1991; 87: 219-27.

2. Factor-Litvak P, Wasserman G, Kline JK, Graziano J. The Yugoslavia prospective study of environmental lead exposure. Environ Health Perspect. 1999; 107: 9-15.

3. Schnaas L, Rothenberg SJ, Perroni E, Martinez S, Hernandez C, Hernandez RM. Temporal pattern in the effect of postnatal blood lead level on intellectual development of young children. Neurotoxicol Teratol. 2000; 22: 805-10.

4. Canfield RC, Henderson CR, Cory-Slechta DA, Cox C, Jusko TA, Lanphear BP. Intellectual impairment in children with blood lead concentrations below 10 ?g per deciliter. N Engl J Med. 2003; 348: 1517-26.

5. Needleman HL, Gatsonis CA. Low-level lead exposure and the IQ of children. A meta-analysis of modern studies. JAMA 1990; 263: 673-8.

6. Schwartz J. Low-level lead exposure and children's IQ: a meta-analysis and search for a threshold. Environ Res. 1994; 65: $42-55$

7. Chiodo LM, Jacobson SW, Jacobson JL. Neurodevelopmental effects of postnatal lead exposure at very low levels. Neurotoxicol Teratol. 2004; 26:359-71.
8. Lanphear BP, Hornung R, Khoury J, Yolton K, Baghurst P, Bellinger DC, et al. Low-level environmental lead exposure and children's intellectual function: an international pooled analysis. Environ Health Perspect. 2005; 113: 894-9.

9. Rothenberg SJ, Rothenberg JC. Testing the dose-response specification in epidemiology: public health and policy consequences for lead. Environ Health Perspect. 2005; 113:1190-5.

10. Téllez-Rojo MM, Bellinger DC, Arroyo-Quiroz C, LamadridFigueroa $\mathrm{H}$, Mercado-García A, Schnaas-Arrieta $L$ et al. Longitudinal associations between blood lead concentrations lower than $10 \mathrm{microgram} / \mathrm{dL}$ and neurobehavioral development in environmentally exposed children in Mexico City. Pediatrics. 2006; 118. Available from: http:www.pediatrics.org/cgi/content/full/118/2/e323

11. Bellinger DC, Stiles KM, Needleman HL. Low-level lead exposure, intelligence and academic achievement: a long-term follow-up study. Pediatrics. 1992; 90: 855-61.

12. Leviton A, Bellinger D, Allred EN, Rabinowitz M, Needleman $H$ Schoenbaum S. Pre- and postnatal low-level lead exposure and children's dysfunction in school. Environ Res. 1993; 60: 30-43.

13. Tong S, Baghurst PA, Sawyer MG, Burns J, McMichael AJ. Declining blood levels and changes in cognitive function during childhood: the Port Pirie cohort study. JAMA 1998; 280: 1915-19. 
14. Chen A, Dietrich KN, Ware JH, Radcliffe J, Rogan WJ. IQ and blood lead from 2 to 7 years of age: are the effects in older children the residual of high blood lead concentrations in 2year-olds? Environ Health Perspect. 2005; 113: 597-601.

15. Lanphear BP, Dietrich KN, Auinger P, Cox C. Cognitive deficits associated with blood lead concentrations $<10$ ?g/dL in US children and adolescents. Public Health Rep. 2000; 115: $521-9$.

16. Al-Saleh 1, Nester M, DeVol E, Shinwari N, Munchari L, AlShahria S. Relationships between blood lead concentrations, intelligence, and academic achievement of Saudi Arabian schoolgirls. Int J Hyg Environ Health. 2001; 204: 165-74.

17. Walkowiak J, Altmann L, Kramer U, Sveinsson K, Turfeld M, Weishoff Houben M, et al. Cognitive and sensorimotor functions in 6-year-old children in relation to lead and mercury levels: adjustment for intelligence and contrast sensitivity in computerized testing. Neurotoxicol Teratol. 1998; 20: 511-21.

18. Needleman HL. Lead poisoning. Annu Rev Med. 2004; 55 : 209-22.

19. Dietrich KN, Ris MD, Succop PA, Berger OG, Bornschein RL. Early exposure to lead and juvenile delinquency. Neurotoxicol Teratol. 2001; 23: 511-8.
20. Bellinger D, Leviton A, Allred E, Rabinowitz M. Pre- and postnatal lead exposure and behavior problems in school-aged children. Environ Res. 1994; 66: 12 -30.

21. Burns JM, Baghurst PA, Sawyer MG, McMichael AJ, Tong SL. Lifetime low-level exposure to environmental lead and children's emotional and behavioral development at ages 11-13 years. The Port Pirie Cohort Study. Am J Epidemiol. 1999; 149: $740-9$.

22. Chen A, Cai B, Dietrich KN, Radcliffe J, J. Rogan WJ. Lead Exposure, IQ, and Behavior in Urban 5- to 7-Year-Olds: Does Lead Affect Behavior Only by Lowering IQ? Pediatrics 2007; 119: $650-8$.

23. Binns HJ, Campbell C, Brown MJ Interpreting and managing blood lead levels of less than 10 ?g/dL in children and reducing childhood exposure to lead: Recommendations of the Centers for Disease Control and Prevention Advisory Committee on Childhood Lead Poisoning Prevention. Pediatrics, 2007; 120: 1285-1298.

24. Rosner D, Markowitz G. Standing Up to the Lead Industry: An Interview with Herbert Needleman. Public Health Rep 2005; 120: $330-337$. 